\title{
A Method for Computing the Circular Coverage Function
}

\author{
By A. R. DiDonato and M. P. Jarnagin
}

1. Introduction. In this paper an efficient method is described for the numerical evaluation, with a high-speed digital computer, of a special case of the integral of an uncorrelated bivariate Gaussian distribution centered at the origin over the area of an arbitrarily placed circle in the plane. This function, popularly known as the circular coverage function or as the non-central chi-square distribution for two degrees of freedom*, can be written as

$$
P(R, D) \equiv \frac{1}{2 \pi \sigma_{x} \sigma_{y}} \iint \exp \left\{-\frac{1}{2}\left[\left(\frac{x}{\sigma_{x}}\right)^{2}+\left(\frac{y}{\sigma_{y}}\right)^{2}\right]\right\} d x d y,
$$

where $S$ is the circle: $(x-h)^{2}+(y-k)^{2}=(\sigma R)^{2}$, where $\sigma_{x}=\sigma_{y}={ }^{\prime} \sigma$, and $\sigma D$ is the radial distance from the origin to the center $(h, k)$ of the circle of integration, $S$. Because of the equivalence mentioned above, a great deal of published literature applies. The papers [13], [15], suggested by the referee, list a large number of such references.

The average computing time for the calculation of the integral in equation (1) to six decimal digits, by the method of this paper, is six milliseconds on the IBM 7090 and ten milliseconds on NORC. An extensive inverse table, which is described in the last section of this paper and which is given in [4], has been computed with $R$ as a function of $P$ and $D$. A condensed version, Table 1 , is presented herein.

In the general case [3], [11] suppose the uncorrelated bivariate Gaussian distribution centered at the origin of an Oxy Cartesian coordinate system has standard deviations $\sigma_{x}, \sigma_{y}$ along the $x$ and $y$ axes respectively, and that the integral of this function is to be evaluated over a circle of radius $\bar{R}$ with center at $(h, k)$. Then the probability, $P$, can be written in polar coordinates accordingly:

$$
\begin{aligned}
P\left(\frac{\bar{R}}{\sigma_{x}}, \frac{\bar{R}}{\sigma_{y}}, \frac{h}{\sigma_{x}}, \frac{k}{\sigma_{y}}\right)= & \frac{1}{2 \pi \sigma_{x} \sigma_{y}} \int_{0}^{\bar{R}} \int_{0}^{2 \pi} \\
& \cdot \exp \left\{-\frac{1}{2}\left[\left(\frac{h+r \cos \theta}{\sigma_{x}}\right)^{2}+\left(\frac{k+r \sin \theta}{\sigma_{y}}\right)^{2}\right]\right\} r d r d \theta,
\end{aligned}
$$

where $x-h=r \cos \theta, y-k=r \sin \theta, 0 \leqq r \leqq \bar{R}, 0 \leqq \theta \leqq 2 \pi$.

If

$$
h=k=0 \text {, }
$$

a special case identified as the $V(K, c)$ or elliptical normal probability function (sometimes known by other titles, for example, the generalized circular error function) [4], [5], [6], [10], [14], [15], [16], [18] follows, i.e.,

$$
P\left(\frac{\bar{R}}{\sigma_{x}}, \frac{\bar{R}}{\sigma_{y}}, 0,0\right) \equiv V(K, c)=\frac{1}{c} \int_{0}^{K} \exp \left(-\frac{B}{2} r^{2}\right) I_{0}\left(\frac{A r^{2}}{2}\right) r d r
$$

Received July 27, 1961.

* The equivalence between the function $P(R, D)$ of equation (1) and the non-central chisquare distribution is evident from equation (2) in [13]. 
where

$$
0 \leqq c \equiv \frac{\sigma_{y}}{\sigma_{x}} \leqq 1, \quad K \equiv \bar{R} / \sigma_{x}, \quad A \equiv \frac{1-c^{2}}{2 c^{2}}, \quad B \equiv \frac{1+c^{2}}{2 c^{2}} .
$$

$I_{0}(x)$ is the modified Bessel function of the first kind of order zero, [8]. Equation (3) is derived by setting $h=k=0$ in equation (2), by using the trigonometric identity $1( \pm) \cos 2 \theta=2\left(\begin{array}{c}\cos ^{2} \theta \\ \sin ^{2} \theta\end{array}\right)$, and by introducing an integral expression for $I_{0}(x)$ which is given by

$$
I_{0}(x)=\frac{1}{\pi} \int_{0}^{\pi} \exp (-x \cos \theta) d \theta .
$$

Equation (4) can be derived from Example 1 (ii), page 62, in [8].

If

$$
\sigma_{x}=\sigma_{\dot{y}}=\sigma,
$$

in equation (2), the distribution is circular normal. In this case, in which $h$ and $k$ are arbitrary, the center of the circle of integration can always be taken as offset a distance of $\sigma D$ from the origin along the positive $x$ axis by simply introducing a rotation of axes through the angle arc $\tan \left(\frac{k}{h}\right)$. Moreover, by introducing the integral expression for $I_{0}(x)$ as given by equation (4), the circular coverage function, $P(R, D)$, [1], [4], [6], [7], [9], [12], [13], [14], [17], is obtained from equation $(2)$, i.e.,

$$
P\left(\frac{\bar{R}}{\sigma_{x}}, \frac{\bar{R}}{\sigma_{x}}, \frac{h}{\sigma_{x}}, \frac{k}{\sigma_{x}}\right) \equiv P(R, D)=\exp \left(-D^{2} / 2\right) \int_{0}^{R} \exp \left(-r^{2} / 2\right) I_{0}(r D) r d r,
$$

where $R \equiv \bar{R} / \sigma_{x}, D^{2} \equiv\left(h^{2}+k^{2}\right) / \sigma_{x}{ }^{2}$.

The function $\partial P(R, D) / \partial R$ is required for computing the inverse function, $R(P, D)$, by the Newton-Raphson procedure (Appendix C, [4]) and is also of use in computing $P(R, D)$ itself (see equation (9)). This function is obtained straightforwardly from equation (5) as

$$
\frac{\partial P}{\partial R}=R \exp \left(-\frac{R^{2}+D^{2}}{2}\right) I_{0}(R D) .
$$

It is apparent by comparing equations (6), (9) that $\partial P / \partial R$ can be computed simultaneously with $P(R, D)$.

In a previous paper, [18], a very efficient computing method was described for calculation of the $V(K, c)$ function. The success of the method warranted consideration of extending the technique to the $P(R, D)$ function. This is not as straightforward as for $V(K, c)$; nevertheless, it is easily possible because of the existence of a simple functional relationship, equation (9), between $P(R, D)$ and $V(K, c)$.

2. The Relationship between $P(R, D)$ and $V(K, c)$. The relationship between $P$ and $V$ can be derived by utilizing two preliminary results which are given by Fettis, in terms of $q \equiv 1-P$, in equations (I-35) and (I-44) in [6]. They can be 
stated in terms of $P$ as:

$$
\begin{array}{ll}
P(R, D)-P(D, R)= \pm V\left(|R-D|, \frac{|R-D|}{R+D}\right) & \begin{array}{l}
(+) \text { if } R>D \\
(-) \text { if } R<D
\end{array} \\
P(R, D)+P(D, R)=1-\exp \left(-\frac{R^{2}+D^{2}}{2}\right) I_{0}(R D)
\end{array}
$$

Equation (8) is easily derived. The origin of equation (7) is not known to the authors. The referee has pointed out that a geometrical proof was given by Dr. David C. Kleinecke of the University of California in 1955. (See also paper I of [15], page 613). Mr. Fettis has kindly placed at the disposal of the authors some correspondence which indicates that the relationship was given in a Sandia Corporation working paper in 1952, and that it was believed to have been originally derived in a British publication by using power series.

It follows by adding the corresponding sides of equations (7) and (8) that*

$$
\begin{array}{r}
P(R, D)=\frac{1}{2}\left[1-\exp \left(-\frac{R^{2}+D^{2}}{2}\right) I_{0}(R D) \pm V\left(|R-D|, \frac{|R-D|}{R+D}\right)\right] \\
(+) \text { if } R>D \\
(-) \text { if } R<D .
\end{array}
$$

Thus, the $P(R, D)$ function is computable at virtually the same speed as $V(K, c)$, since the second term in the brackets turns out to be a by-product of the recurrence relations which are used to compute $V$ in the last term. Consequently, if there exists a satisfactory computing program for the $V$ function, a computing program of equal merit can be realized for the $P(R, D)$ function.

3. Recurrence Relations. The $V$ function that appears as the last term of equation (9) is identified with equation (3) by setting

$$
K=|R-D|, \quad c=|R-D| /(R+D) .
$$

It follows that

$$
A=\frac{2 R D}{(R-D)^{2}}, \quad B=\frac{R^{2}+D^{2}}{(R-D)^{2}}
$$

where it is assumed $R \neq D$. If $R=D$, then, from equation (7), $V(|R-D|$, $\left.\frac{|R-D|}{R+D}\right)$ vanishes and $P(R, D)$ is given by the first two terms of equation (9).

The two series representations for $V\left(|R-D|, \frac{|R-D|}{R+D}\right)$ from which the basic recurrence relations are derived are given by:

* Guenther recently (see equation (2) in [9]) derived an equation for $P(R, D)$ in terms of $I_{0}(x)$ and the incomplete gamma function, which can be shown to be equivalent to equation (9) of the present paper. However, he did not exploit his relationship from the point of view of developing an efficient program for a high-speed digital computer. 
(10)

$$
\begin{aligned}
& V\left(|R-D|, \frac{|R-D|}{R+D}\right)= \frac{\left|R^{2}-D^{2}\right|}{R D} \sum_{n=0}^{\infty}\left(\frac{1}{n !}\right)^{2} \\
& \cdot \int_{0}^{R D / 2} \exp \left(-\frac{R^{2}+D^{2}}{R D} w\right) w^{2 n} d w \equiv \sum_{n=0}^{\infty} T_{2 n}, \\
& V\left(|R-D|, \frac{|R-D|}{R+D}\right)= 1-\frac{\left|R^{2}-D^{2}\right|}{4 R D \sqrt{\pi}} \sum_{n=0}^{N} \frac{[(2 n) !]^{2}}{2^{4 n}(n !)^{3}} \\
& \cdot \int_{2 R D}^{\infty} \exp \left[-\frac{(R-D)^{2}}{4 R D} w\right] w^{-\left(\frac{2 n+1}{2}\right)} d w=1-\sum_{n=0}^{N} M_{2 n+1} .
\end{aligned}
$$

The detailed derivations of equations (10), (11) are given in [4]. Briefly, to obtain equation (10), introduce a variable of integration transformation

$$
w=A r^{2} / 4
$$

into the integral of equation (3), then replace $I_{0}(2 w)$ by its Taylor series expansion (see page 14, [8]),

$$
I_{0}(2 w)=\sum_{n=0}^{\infty}\left(\frac{1}{n !}\right)^{2}\left(\frac{2 w}{2}\right)^{2 n}
$$

which is convergent for all values of $w$, and subsequently reverse the order of integration and summation, which can be justified by application of the Weierstrass " $M$ " test. In order to derive equation (11) introduce a variable of integration transformation

$$
w=A r^{2}
$$

into the integral of equation (3) and use the fact that

$$
\frac{1}{2 A c} \int_{0}^{\infty} \exp \left(-\frac{B w}{2 A}\right) I_{0}\left(\frac{w}{2}\right) d w=1
$$

(See page 76, [8]). In the resulting integral expression, call it $J$, with upper and lower limits of integration of infinity and $A K^{2}$ respectively, replace $I_{0}\left(\frac{w}{2}\right)$ by its asymptotic expansion (see page $58,[8]$ ), i.e.,

$$
I_{0}\left(\frac{w}{2}\right) \approx \frac{\exp (w / 2)}{\sqrt{2 \pi(w / 2)}} \sum_{n=0}^{N} \frac{[(2 n) !]^{2}}{2^{4 n}(n !)^{3}}(2 w / 2)^{-n},
$$

which is valid for sufficiently large $w$ and finite $N$; subsequently interchange the order of integration and summation. The interchange is justified for all values of $(2 R D)$ for which equation (16) is valid because of the existence of the integral $J$ (see page 17, [2]).

The substitution of equations (13), (16) into equation (6) gives analogous series representations for $\partial P / \partial R$, i.e.,

$$
\begin{gathered}
\frac{\partial P}{\partial R}=R \sum_{n=0}^{\infty} \bar{S}_{2 n}, \\
\frac{\partial P}{\partial R} \approx R\left(\frac{1}{4 R D} \sum_{n=0}^{N} \bar{X}_{2 n+1}\right),
\end{gathered}
$$


where

$$
\begin{array}{cc}
\bar{S}_{2 n} \equiv \exp \left(-\frac{R^{2}+D^{2}}{2}\right)\left(\frac{1}{n !}\right)^{2}\left(\frac{R D}{2}\right)^{2 n}, & n \geqq 0, \\
\bar{X}_{2 n+1} \equiv \frac{2}{\sqrt{\pi}} \exp \left[-\frac{(R-D)^{2}}{2}\right] \frac{[(2 n) !]^{2}}{2^{4 n}(n !)^{3}}(2 R D)^{-\left(\frac{2 n-1}{2}\right),} & n \geqq 0,
\end{array}
$$

following the notation of [4], in which there are slight distinctions between $\bar{S}_{2 n}$, $\bar{X}_{2 n+1}, \bar{Y}_{2 n-1}$, and the corresponding unbarred variables used with $V(K, c)$ and $\partial V / \partial K$.

Thus two schemes are used to compute $P$. If

$$
2 R D \leqq M \quad(M \text { is a positive constant }),
$$

then with reference to equations (10) and (17)

$$
\begin{array}{cc}
T_{2 n}=\left(\frac{2 n-1}{2 n}\right)\left(\frac{2 R D}{R^{2}+D^{2}}\right)^{2} & T_{2 n-2} \\
-\frac{\left|R^{2}-D^{2}\right|}{R^{2}+D^{2}}\left(1+\frac{4 n}{R^{2}+D^{2}}\right) \bar{S}_{2 n}, & n \geqq 1, \\
\bar{S}_{2 n}=\left(\frac{R D}{2 n}\right)^{2} \bar{S}_{2 n-2}, & n \geqq 1,
\end{array}
$$

where the necessary initial terms are given by

$$
\begin{gathered}
T_{0}=\frac{\left|R^{2}-D^{2}\right|}{R^{2}+D^{2}}\left[1-\exp \left(-\frac{R^{2}+D^{2}}{2}\right)\right]=\frac{\left|R^{2}-D^{2}\right|}{R^{2}+D^{2}}\left(1-\bar{S}_{0}\right), \\
\bar{S}_{0}=\exp \left(-\frac{R^{2}+D^{2}}{2}\right) .
\end{gathered}
$$

The following brief comments are made on the derivation of recurrence relations (22) and (23). Fuller details are given in [4]. From equations (13) and (19), $\bar{S}_{2 n}$ is the general term in the series obtained by multiplying every term of the Taylor series for $I_{0}(R D)$ by $\exp \left[-\left(R^{2}+D^{2}\right) / 2\right]$, and equations (23) and (25) are obtained immediately. If $T_{2 n}$ is regarded as defined by equation (10), two successive integrations by parts give $T_{2 n}$ in terms of $T_{2 n-2}, R, D$, and $n$, after which the term not containing $T_{2 n-2}$ can be written more concisely in terms of $\bar{S}_{2 n}$, and equation (22) is the result.

These basic recurrence relations are cycled until

$$
T_{2 n}<\epsilon, \quad \bar{S}_{2 n}<\epsilon,
$$

$$
(\epsilon>0) \text {. }
$$

Then $P$ and $\partial P / \partial R$ are given correctly to at least $\left(\left|\log _{10} \epsilon\right|-1\right)$ decimal digits by

$$
\begin{aligned}
& P(R, D) \approx \frac{1}{2}\left[1-\sum_{n=0}^{N^{\prime}} \bar{S}_{2 n} \pm \sum_{n=0}^{N^{\prime}} T_{2 n}\right] \quad \begin{array}{l}
(+) \text { if } R>D \\
(-) \text { if } R<D,
\end{array} \\
& \frac{\partial P}{\partial R} \approx R \sum_{n=0}^{N^{\prime}} \bar{S}_{2 n} \text {. }
\end{aligned}
$$

If it is assumed that

$$
2 R D>M
$$


then with reference to equations (11) and (18)

$$
\begin{array}{rlrl}
M_{2 n+1}=\frac{\left|R^{2}-D^{2}\right|}{4 R D} \bar{Y}_{2 n-1}-\frac{(R-D)^{2}}{4 R D}\left(\frac{2 n-1}{2 n}\right) M_{2 n-1}, & & n \geqq 1, \\
\bar{Y}_{2 n-1}=\frac{1}{4 R D}\left(\frac{2 n-1}{2 n}\right) \bar{X}_{2 n-1}, & n \geqq 1, \\
\bar{X}_{2 n+1}=(2 n-1) \bar{Y}_{2 n-1}, & n \geqq 1,
\end{array}
$$

where the initial terms are given by

$$
\begin{aligned}
M_{1}= & \frac{1}{\sqrt{2 R D}}\left(\frac{R+D}{\sqrt{2}}\right) \frac{2}{\sqrt{\pi}} \int_{\frac{|R-D|}{\sqrt{2}}}^{\infty} \exp \left(-y^{2}\right) d y \\
= & \frac{1}{\sqrt{2 R D}}\left(\frac{R+D}{\sqrt{2}}\right)\left[1-\operatorname{Erf}\left(\frac{|R-D|}{\sqrt{2}}\right)\right] \\
& \bar{X}_{1}=\sqrt{2 R D} \frac{2}{\sqrt{\pi}} \exp \left[-\frac{(R-D)^{2}}{2}\right] .
\end{aligned}
$$

The following brief comments are made on the derivation of equations (30) to (33). Fuller details are given in [4]. From equations (16) and (20), $\bar{X}_{2 n+1}$ is the general term in the expansion obtained by multiplying every term of the asymptotic expansion of $I_{0}(R D)$ by $4 R D \exp \left[-\left(R^{2}+D^{2}\right) / 2\right]$. Equations (31) and (32), which together form a recurrence relation generating $\bar{X}_{2 n+1}$, are obtained immediately, the introduction of the variable $\bar{Y}_{2 n-1}$ leading to a computationally efficient algorithm for the simultaneous evaluation of the last two terms in equation (9). If $M_{2 n+1}$ is regarded as defined by equation (11), an integration by parts gives $M_{2 n+1}$ in terms of $M_{2 n-1}, R, D$, and $n$, after which the term not containing $M_{2 n-1}$ can be written more concisely in terms of $\bar{Y}_{2 n-1}$, and recurrence relation (30) is the result. $M_{1}$, originally obtained by putting $n=0$ in the definition of $M_{2 n+1}$, is expressed in equation (33) in terms of the error function (see [3], equations (6)) by a transformation in which $y$ is $\left(\frac{1}{2}\right)|R-D| \sqrt{w /(R D)}$.

These basic recurrence relations are cycled until

$$
M_{2 n+1}<\epsilon, \quad \bar{X}_{2 n+1}<\epsilon,
$$

Then $P(R, D)$ and $\partial P / \partial R$ are given correctly to $\left(\left|\log _{10} \epsilon\right|-1\right)$ decimal digits by

$$
\begin{gathered}
P(R, D) \approx \frac{1}{2}\left[1-\frac{1}{4 R D} \sum_{n=0}^{N} \bar{X}_{2 n+1} \pm\left(1-\sum_{n=0}^{N} M_{2 n+1}\right)\right] \begin{array}{l}
(+) \text { if } R>D \\
(-) \text { if } R<D,
\end{array} \\
\frac{\partial P}{\partial R} \approx R\left[\frac{1}{4 R D} \sum_{n=0}^{N} \bar{X}_{2 n+1}\right] .
\end{gathered}
$$

The determination of the constant $M$ is discussed in Appendix A of [4]. If the constants $M$ and $\epsilon$ were chosen such that

$$
M=30, \quad \epsilon=10^{-8},
$$

then sufficient tests were made on the results to assure seven-decimal digit accuracy in the values of $P$ and $\partial P / \partial R$ for all values of $R$ and $D$. The tests are described in [4]. The maximum number of terms, $N^{\prime}$, required for seven-decimal 
digit accuracy in either series that occurs in equation (27) was twenty for $0<R \leqq$ $126,0 \leqq D \leqq 120$.

4. Table Computation-Discussion of Results. The extensive inverse table, mentioned in the introduction, has $R$ tabulated as a function of $P$ and $D$ for the

TABLE 1

Inverse $P(R, D)$ Table, $R=R(P, D)$

\begin{tabular}{|c|c|c|c|c|c|c|c|c|}
\hline $\begin{array}{l}D \\
P\end{array}$ & 0.1 & 0.5 & 1.0 & 1.5 & 2.0 & 3.0 & 4.0 & 5.0 \\
\hline .01 & 0.142132 & 0.150917 & 0.181965 & 0.247976 & 0.377894 & 0.973968 & 1.857355 & 2.807007 \\
\hline .05 & 0.321093 & 0.340911 & 0.410355 & 0.552995 & 0.803492 & 1.589932 & 2.514287 & 3.475659 \\
\hline .10 & 0.460192 & 0.488541 & 0.586808 & 0.780875 & 1.090931 & 1.931431 & 2.867729 & 3.833372 \\
\hline .15 & 0.571548 & 0.606683 & 0.727145 & 0.956651 & 1.299471 & 2.164629 & 3.107065 & 4.075094 \\
\hline .20 & 0.669719 & 0.710800 & 0.850071 & 1.106744 & 1.470965 & 2.351156 & 3.297689 & 4.267393 \\
\hline .25 & 0.760426 & 0.806964 & 0.962923 & 1.241576 & 1.621141 & 2.511865 & 3.461479 & 4.432486 \\
\hline .30 & 0.846714 & 0.898407 & 1.069594 & 1.366651 & 1.757905 & 2.656649 & 3.608743 & 4.580828 \\
\hline .35 & 0.930528 & 0.987190 & 1.172547 & 1.485396 & 1.885955 & 2.791156 & 3.745340 & 4.718356 \\
\hline .40 & 1.013296 & 1.074827 & 1.273564 & 1.600226 & 2.008448 & 2.919061 & 3.875068 & 4.848912 \\
\hline .45 & 1.096204 & 1.162568 & 1.374100 & 1.713036 & 2.127745 & 3.043037 & 4.000676 & 4.975274 \\
\hline .50 & 1.180355 & 1.251580 & 1.475479 & 1.825472 & 2.245802 & 3.165246 & 4.124378 & 5.099676 \\
\hline .55 & 1.266891 & 1.343064 & 1.579042 & 1.939121 & 2.364426 & 3.287634 & 4.248157 & 5.224119 \\
\hline .60 & 1.357113 & 1.438388 & 1.686286 & 2.055680 & 2.485472 & 3.412162 & 4.374006 & 5.350606 \\
\hline .65 & 1.452637 & 1.539246 & 1.799042 & 2.177146 & 2.611062 & 3.541034 & 4.504154 & 5.481380 \\
\hline .70 & 1.555634 & 1.647914 & 1.919739 & 2.306101 & 2.743883 & 3.677012 & 4.641388 & 5.619238 \\
\hline .75 & 1.669270 & 1.767705 & 2.051892 & 2.446209 & 2.887695 & 3.823927 & 4.789566 & 5.768053 \\
\hline .80 & 1.798604 & 1.903913 & 2.201075 & 2.603222 & 3.048351 & 3.987718 & 4.954663 & 5.933817 \\
\hline .85 & 1.952745 & 2.066052 & 2.377281 & 2.787369 & 3.236215 & 4.178871 & 5.147218 & 6.127099 \\
\hline .90 & 2.151322 & 2.274618 & 2.601947 & 3.020515 & 3.473382 & 4.419704 & 5.389656 & 6.370384 \\
\hline .95 & 2.453851 & 2.591661 & 2.939763 & 3.368463 & 3.826253 & 4.777225 & 5.749279 & 6.731139 \\
\hline .97 & 2.654829 & 2.801806 & 3.161592 & 3.595668 & 4.056141 & 5.009727 & 5.982997 & 6.965523 \\
\hline .99 & 3.042407 & 3.205999 & 3.584494 & 4.026818 & 4.491533 & 5.449368 & 6.424667 & 7.408327 \\
\hline 995 & 3.263342 & 3.435790 & 3.823110 & 4.269216 & 4.735933 & $\mathbf{5 . 6 9 5 8 2 6}$ & 6.672133 & 7.656366 \\
\hline 999 & 3.726147 & 3.915765 & 4.318250 & 4.770776 & 5.240984 & 6.204548 & 7.182694 & 8.167991 \\
\hline 9999 & 4.302554 & 4.511127 & 4.927840 & 5.386401 & 5.860000 & 6.827233 & 7.807274 & 8.793692 \\
\hline .99999 & 4.810368 & 5.033640 & 5.459903 & 5.922582 & 6.398559 & 7.368429 & 8.349868 & 9.337129 \\
\hline .999999 & 5.269458 & 5.504595 & 5.937784 & 6.403513 & 6.881283 & 7.853179 & 8.835714 & 9.823646 \\
\hline$\frac{D}{P}$ & 6.0 & 8.0 & .0 & 2 & 30.0 & 5 & 8 & 120.0 \\
\hline .01 & 3.778556 & 5.747335 & 7.7 & 22 & 100 & 89 & 999 & $\mid 117.6779$ \\
\hline .05 & 4.452164 & 6.424982 & 8.4 & 18. & 28.3 & 48. & 78. & 118 \\
\hline .10 & 4.811875 & 6445 & 8.771 & 18. & 28.7 & 48.72858 & 78.72475 & 118.7226 \\
\hline .15 & 5.054765 & 7.030393 & 9.016299 & 18.98923 & 28.98053 & 48.97367 & 78.96986 & 118.9678 \\
\hline .20 & 5.247904 & 7.224314 & 9.210559 & 19.18391 & 29.17528 & 49.16846 & 79.1 & 119.1626 \\
\hline .25 & 5.413665 & 7.390705 & 9.377228 & 19.35094 & 29.34237 & 3558 & 179 & 11 \\
\hline .30 & 5.562570 & 7.540148 & 9.526912 & 19.50093 & 29.4 & 49. & 79. & 11 \\
\hline .35 & 590 & 7.6 & 9.66 & 19. & 29.63145 & 49.62472 & 79.62094 & 119.6189 \\
\hline .40 & 5.831589 & 7.810077 & 9.797253 & 19.77181 & 29.76339 & 49.75668 & 79.75291 & 119. \\
\hline .45 & 5.958359 & 7.937251 & 9.924613 & 19.89941 & 29.89104 & 49.88435 & 79.88059 & \\
\hline .50 & 6.083144 & 8.0 & 10.0 & 20.02499 & 30.01667 & 50. & 80.0 & 12 \\
\hline .55 & 7953 & 8.1 & 10.1 & 20.15058 & 30.14229 & 50.13565 & 80.13191 & 120.1298 \\
\hline .60 & 6.334797 & 8.314803 & 10.30269 & 20.27819 & 30.26994 & 50.26332 & 80.25959 & 120.2575 \\
\hline .65 & 6.465923 & 8.4 & 10.43434 & 20.41008 & 30.4 & 39528 & 80.39156 & 895 \\
\hline .70 & 6.604135 & 8. & 10.5 & 20.5 & 92 & & 63 & 120. \\
\hline .75 & 6.753314 & 8.7 & 10.722 & 20.6 & 30.6 & 42 & 80.68071 & 120.6786 \\
\hline $80-2>$ & 6.919464 & 8.9 & 10.88959 & 20.86610 & 30.85806 & 154 & 1784 & 120. \\
\hline .85 & 7.113172 & 9.0 & 11.08398 & 21.06080 & 31.05282 & 633 & 81.04264 & 121.0406 \\
\hline .90 & 7.356958 & & 11.3 & 21.30578 & 31.29787 & & & 121.2857 \\
\hline .95 & 7.718391 & 9.7 & 11.6 & 21.66 & 31.66108 & 69 & 81.65104 & 121.6490 \\
\hline .97 & 7.953181 & 9.9 & 11.92658 & 21.90468 & 31.89696 & 51.89061 & 81.88697 & 121.8849 \\
\hline .99 & 8.396685 & 10.38117 & 12.37128 & 22.34999 & 32.34240 & 52.33612 & 82.33251 & 122.3305 \\
\hline 995 & & & 12.62029 & 22.59934 & 32.59182 & 52.58558 & 82.58198 & 122.5800 \\
\hline 999 & & 11.14304 & 13.13378 & 23.11348 & 33.10609 & 53.09994 & 83.09636 & 123.0943 \\
\hline 999 & 9.783802 & 11.77031 & 13.76151 & 23.74194 & 33.73473 & 53.72866 & 83.72513 & 123.7231 \\
\hline 99999 & 10.32779 & 12.31496 & 14.30652 & 24.28755 & 34.28047 & 54.27449 & 84.27098 & 124.2690 \\
\hline .999999 & 10.81475 & 12.80245 & 14.79431 & 24.77585 & 34.76889 & 54.76298 & 84.75950 & 124.7575 \\
\hline
\end{tabular}


following ranges:

$$
\begin{aligned}
& P=0.01(.01) 0.99 \\
& D=0(.1) 5(.2) 10(2) 20(5) 120
\end{aligned}
$$

and

$$
\begin{aligned}
& P=.99(.0005) .9990(.0001) .9999(.00001) .99999(.000001) .999999 \\
& D=0, .05, .10, .25, .75,1,1.5,2,3,4,5,6,8,10,20,30,50,80,120
\end{aligned}
$$

This table required the calculation of over $45,000 P(R, D)$ functions to an accuracy of seven or more decimal digits. The tabulated values of $R$, determined by a NewtonRaphson process, are correct to within one unit in the last digit position given. The method by which this conclusion was verified is given in Appendix C of [4]. A condensed version of the complete table is given below. The complete table as well as a similar one for $K$ as a function of $V$ and $c$ are available by direct request to the authors.

It can be proved that $R(P, D)$ as a function of $P$ approximates a univariate normal distribution to any desired accuracy for sufficiently large fixed values of $D$ and $|R-D| /(R+D) \ll 1$. The relation between $R$ and $P$ in this case is given by

$$
P(R, D) \approx \frac{1}{2}\left[1+\operatorname{Erf}\left(\frac{R-\mu_{R}}{\sqrt{2}}\right)\right]=\frac{1}{\sqrt{2 \pi}} \int_{-\infty}^{R-\mu_{R}} e^{-t^{2} / 2} d t
$$

where $\mu_{R} \equiv R(0.50, D) \approx D+1 /(2 D)$. (A slightly different formulation of the asymptotic behavior was given by Germond in [7]). This shows that the functional relationship is symmetric with respect to the point $R=\mu_{R}, P=0.50$. This is evident from a study of Table 1. Also, if $20 \leqq D \leqq 25$, and if $\mu_{R}$ is computed from the approximation $D+1 /(2 D)$ (which for these values of $D$ is accurate to $10^{-5}$ or better), and if values of $R$ as a function of $P$ are then computed from equation (39) by inverse interpolation in an error function or univariate probability integral table, the results are, in general, correct within $10^{-3}$, or one unit in the fifth significant figure of $R$. Further, the accuracy improves rapidly as $D$ increases. This means that an efficient inverse table such as Table 1 need extend only from $P=0$ to $P=0.50$ if $D$ is large. Each value of $R$ for $P>0.50$ is then found with only one subtraction and one addition by using the symmetry property stated above.

5. Acknowledgment. The authors wish to thank Mr. David Eliezer and Mr. Robert Belsky, who programmed and coded the editing procedure for setting up the complete tables, and Mr. Robert Gramp, who programmed and coded the method of computing $V(K, c)$ and $P(R, D)$ for the IBM 7090. The authors are indebted to the referee for suggestions which materially improved the introductory portion of this paper, for correcting a false impression the authors had concerning the origin of equation (9), and for calling the attention of the authors to the univariate normal character of the circular coverage function for large $D$, as commented on at the end of Section 4.

U. S. Naval Weapons Laboratory

Dahlgren, Virginia 
1. H. E. DANiELS, "The covering circle of a sample from a circular normal distribution," Biometrika, v. 39,1952 , p. 137-143.

2. N. G. DE Bruisn, Asymptotic. Methods in Analysis, North-Holland Publishing Co., Amsterdam, and Interscience Publishers, Inc., New York, 1958.

3. A. R. DiDonato \& M. P. JaRnagin, "Integration of the general bivariate Gaussian distribution over an offset circle," Math. Comp., v. 15, 1961, p. 375-382.

4. A. R. DiDonato \& M. P. JaRnagin, A Method for Computing the Generalized Circular Error Function and the Circular Coverage Function, NWL Report 1768, U. S. Naval Weapons Laboratory, Dahlgren, Virginia, 23 January 1962.

5. R. V. Esperti, Tables of the Elliptical Normal Probability Function, Defense Systems Division, General Motors Corporation, Warren, Michigan, 6 April 1960.

6. H. E. Fetris, Some Mathematical Identities and Numerical Methods Relating to the Bivariate Normal Probability for Circular Regions, WADC Technical Note 57-383, ASTIA Document No. AD142135, Wright Air Development Center, Wright-Patterson Air Force Base, Ohio, December, 1957.

7. H. H. Germond, The Circular Coverage Function, RAND Corporation Research Memorandum RM-330, 26 January 1950.

8. A. Gray, G. B. Mathews \& T. M. MacRobert, A Treatise on Bessel Functions and Their Applications to Physics, Second Edition, The Macmillan Co., New York and London, 1922.

9. W. C. Guenther, "Circular probability problems," Amer. Math. Monthly, v. 68, n. 6, 1961 , p. 541-544.

10. H. L. Harter, “Circular error probabilities," J. Amer. Statist. Assoc., v. 55, n. 292, 1960 , p. $723-731$.

11. J. R. Lowe, "A table of the bivariate normal distribution over an offset circle," $J$. Roy. Statist. Soc., Ser. B, v. 22, 1960, p. 177-186.

12. Offset Circle Probabilities, RAND Corporation Report R-234, 14 March 1952.

13. P. B. Patnalk, "The non-central $\chi^{2}$ - and $F$-distributions and their applications," Biometrika, v. 36, 1949, p. 202-232.

14. Probability-of-Damage Problems of Frequent Occurrence, OEG Study 626, Operations Evaluation Group, Office of the Chief of Naval Operations, 11 December 1959.

15. H. RUBEN, "Probability content of regions under spherical normal distributions"; I, Ann. Math. Statist., v. 31, 1960, p. 598-618; II, ibid., v. 31, 1960, p. 1113-1121; III, ibid., v. 32,1961, p. 171-186.

16. H. Solomon, Distribution of Quadratic Forms-Tables and Applications, Applied Mathematics and Statistics Laboratories Technical Report No. 45, Stanford University, 5 September 1960.

17. Table of Circular Normal Probabilities, Bell Aircraft Corporation Report *02-949-106, June 1956. Reviewed in MTAC, v. 11, 1957, p. 210.

18. H. Weingarten \& A. R. DiDonato, "A table of generalized circular error," Math. Comp., v. 15, 1961, p. 169-173. 